\title{
Upscale energy transfer in thick turbulent fluid layers
}

\author{
H. Xia' ${ }^{1}$ D. Byrne', G. Falkovich ${ }^{2}$ and M. Shats ${ }^{1 \star}$
}

Flows in natural fluid layers are often forced simultaneously at scales smaller and much larger than the depth. For example, the Earth's atmospheric flows are powered by gradients of solar heating: vertical gradients cause three-dimensional (3D) convection whereas horizontal gradients drive planetary scale flows. Nonlinear interactions spread energy over scales ${ }^{1,2}$. The question is whether intermediate scales obtain their energy from a large-scale 2D flow or from a small-scale 3D turbulence. The paradox is that 2D flows do not transfer energy downscale whereas 3D turbulence does not support an upscale transfer. Here we demonstrate experimentally how a large-scale vortex and small-scale turbulence conspire to provide for an upscale energy cascade in thick layers. We show that a strong planar vortex suppresses vertical motions, thus facilitating an upscale energy cascade. In a bounded system, spectral condensation into a box-size vortex provides for a self-organized planar flow that secures an upscale energy transfer.

Turbulence in thin layers is quasi-two-dimensional and supports an inverse energy cascade ${ }^{3}$, as has been confirmed in many experiments in electrolytes ${ }^{4-6}$ and soap films ${ }^{7,8}$. In bounded systems the inverse cascade may lead to a spectral condensation, that is, the formation of a flow coherent over the entire domain ${ }^{4-6}$. One expects that in thick layers the flow is $3 \mathrm{D}$ and there is no inverse energy cascade. Indeed, as has been demonstrated in 3D numerical modelling, when the layer thickness, $h$, exceeds half the forcing scale, $l_{\mathrm{f}}, h / l_{\mathrm{f}}>0.5$, the onset of vertical motions destroys the quasi-two-dimensionality of the turbulence and stops the upscale energy transfer ${ }^{9,10}$.

In this Letter we report new laboratory studies of turbulence in layers that show that a large-scale horizontal vortex, either imposed externally or generated by spectral condensation in turbulence, suppresses vertical motions in thick layers. This leads to a robust inverse energy cascade even in thick layers with $h / l_{\mathrm{f}}>0.5$.

In our experiments, turbulence is generated by the interaction of a large number of electromagnetically driven vortices ${ }^{11-13}$. The d.c. electric current flowing through a conducting fluid layer interacts with the spatially variable vertical magnetic field. The field is produced by an array of 900 magnets placed beneath the fluid cell, the size of which is $0.3 \times 0.3 \mathrm{~m}^{2}$. The flow is visualized using seeding particles, which are suspended in the fluid, illuminated using a horizontal laser slab, and filmed from above. Particle image velocimetry (PIV) is used to derive the turbulent velocity fields. To visualize the vertical flows, a vertical laser slab is used and the particle motion is filmed from the side. To quantify the velocity fluctuations in 3D, a defocusing PIV technique has been developed that allows all three velocity components to be measured ${ }^{14}$.

We use two different configurations: (1) a single layer of electrolyte on a solid bottom, and (2) a layer of electrolyte on top of another layer of a non-conducting heavier liquid,

which substantially decreases friction. For a thin double-layer configuration in the presence of a boundary, a 2D inverse cascade was shown to form a spectral condensate, or the box-size vortex, which dominated the flow in a steady state ${ }^{11-13}$. The statistics of the increment $\delta V_{\mathrm{L}}$ across a distance $r$ of the velocity component parallel to $r$ were measured using hundreds of instantaneous velocity fields ${ }^{11-13}$. The second moment of $\delta V_{\mathrm{L}}$ determines the spectrum, whereas the third moment, $S_{3 \mathrm{~L}}=\left\langle\left(\delta V_{\mathrm{L}}\right)^{3}\right\rangle$, is related in isotropic turbulence to the value and the direction of the energy flux $\varepsilon$ across the scale $r$ :

$$
\varepsilon=-(2 / 3) S_{3 \mathrm{~L}} / r
$$

The sign of $S_{3 \mathrm{~L}}$ thus determines the flux direction: $\varepsilon$ is positive in $3 \mathrm{D}$ (direct cascade) and negative in 2D (inverse cascade). Indeed, positive linear $S_{3 \mathrm{~L}}(r)$ has been observed in quasi-2D turbulence in soap films ${ }^{8}$ and in electrolyte layers ${ }^{1-13}$. For turbulence with the condensate, it is crucial to subtract a coherent flow from the instantaneous velocity fields to recover the correct statistics of the turbulent velocity fluctuations ${ }^{13,15}$. The point is that the velocity differences $\delta V$ contain contributions from the spatially inhomogeneous vortex flow $\delta \bar{V}$, in addition to the contributions from the turbulent velocity fluctuations $\delta \tilde{V}: \delta V=\delta \tilde{V}+\delta \bar{V}$. The vortex contribution then enters the higher-order moments: $\left\langle\delta V^{2}\right\rangle=$ $\left\langle\delta \tilde{V}^{2}\right\rangle+\left\langle\delta \bar{V}^{2}\right\rangle,\left\langle\delta V^{3}\right\rangle \approx\left\langle\delta \tilde{V}^{3}\right\rangle+\left\langle\delta \bar{V}^{3}\right\rangle+3\left\langle\delta \bar{V} \delta \tilde{V}^{2}\right\rangle$, and so on. It has been demonstrated that the presence of the large-scale flows substantially modifies $S_{3 \mathrm{~L}}$ and even affects its sign ${ }^{12,13}$.

We now present the new results, starting with a thick two-layer configuration when the top layer thickness $h_{\mathrm{t}}$ exceeds half the forcing scale $l_{\mathrm{f}}$. The forcing scale in this experiment is $l_{\mathrm{f}}=9 \mathrm{~mm}$, and the thicknesses of the bottom and top layers are $h_{\mathrm{b}}=4 \mathrm{~mm}$ and $h_{\mathrm{t}}=7 \mathrm{~mm}$ respectively. The size of the boundary box is $L=120 \mathrm{~mm}$. Visualization in both horizontal $(x-y)$ and vertical $(z-y)$ planes shows that the flow is substantially $3 \mathrm{D}$ at the early stage of the evolution, as seen in Fig. 1a,b, in agreement with numerics. However, if one waits long enough, one sees the appearance of a large planar vortex, the diameter of which is limited by the box size (numerical simulations ${ }^{10}$ were done on a shorter timescale). Apparently, a residual inverse energy flux, existing even in the presence of $3 \mathrm{D}$ motions, leads to the spectral condensation and to the generation of the large coherent vortex that dominates the flow in a steady state (Fig. 1c). In the vertical cross-section, the flow is now close to planar (Fig. 1d). The vortex gives a strong spectral peak at $k \approx k_{\mathrm{c}}$, as seen in Fig. $1 \mathrm{e}$, where $k_{\mathrm{f}}$ is the forcing wavenumber. The subtraction of the temporal mean reveals the spectrum of the underlying turbulence, see Fig. 1g, which shows a reasonably good agreement with the Kraichnan $k^{-5 / 3}$ inverse energy cascade. Similarly, before the mean subtraction, $S_{3 \mathrm{~L}}$ is negative and is not a linear function of $r$ (Fig. 1f). The subtraction of the mean vortex flow reveals positive $S_{3 \mathrm{~L}}$, which

${ }^{1}$ Research School of Physics and Engineering, The Australian National University, Canberra ACT 0200, Australia, ${ }^{2}$ Physics of Complex Systems, Weizmann Institute of Science, Rehovot 76100, Israel. *e-mail: Michael.Shats@anu.edu.au. 


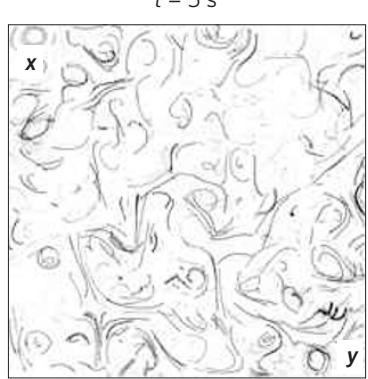

b

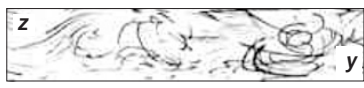

e
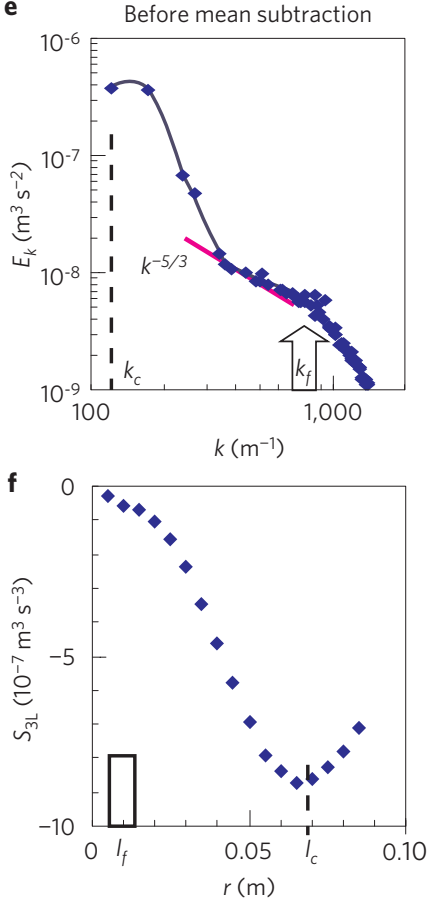

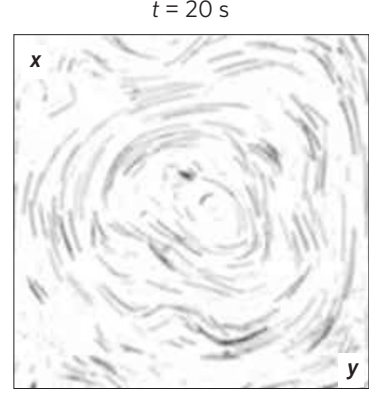

d $z$

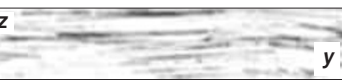

g

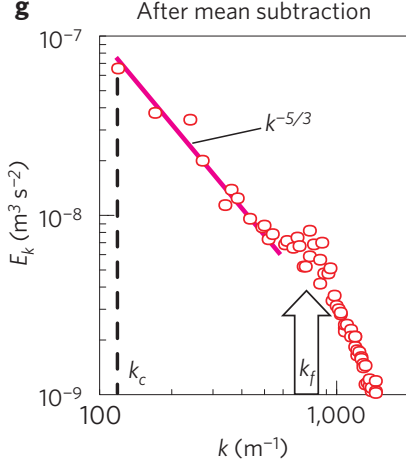

h

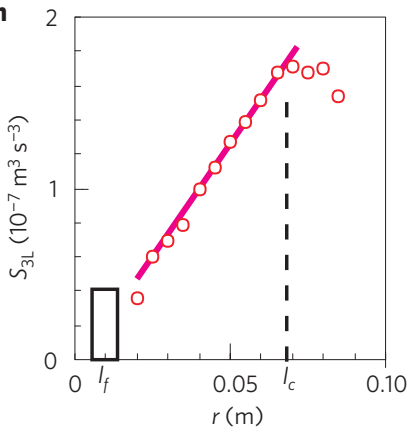

Figure 1 | Structure of turbulence in the double layer configuration. Turbulence is forced electromagnetically by the array of magnetic dipoles, $I_{\mathrm{f}}=9 \mathrm{~mm}$, in the top layer of electrolyte $\left(h_{\mathrm{t}}=7 \mathrm{~mm}\right)$ resting on the $4 \mathrm{~mm}$ layer of non-conducting heavier liquid (Fluorinert). Particle streaks are filmed in the horizontal $x-y$ plane with an exposure time of $2 \mathrm{~s}(\mathbf{a}, \mathbf{c})$ and in the vertical $z-y$ plane with an exposure time of $1 \mathbf{s}(\mathbf{b}, \mathbf{d})$ during the development of the large scale vortex. Images $(\mathbf{a}, \mathbf{b})$ correspond to the time interval centred around $t=5 \mathrm{~s}$ after the flow forcing is switched on; (c,d) correspond to $t=20 \mathrm{~s}$. The size of the horizontal plane box is $120 \mathrm{~mm}$. The spectrum of the steady flow (e) and the third-order structure function $S_{3 L}$ (f) are dominated by the contribution from the large coherent vortex (c), which develops as a result of turbulence spectral condensation. Subtraction of the mean velocity field reveals the spectrum $(\mathbf{g})$ and $S_{3 L}(\mathbf{h})$, which are similar to those in quasi-2D turbulence.

is a linear function of $r$, in agreement with equation (1) (Fig. 1h). The slope of that linear dependence gives the value of the upscale energy flux, $\varepsilon \approx 1.8 \times 10^{-6} \mathrm{~m}^{2} \mathrm{~s}^{-3}$. This value was independently verified by the energy balance analysis, as described in ref 13: $\varepsilon$ is compared with the large-scale flow energy dissipation rate $\varepsilon_{\mathrm{d}}$. For the present experiment this agreement is within $10 \%$.

The results of Fig. 1 indicate that, contrary to expectations, turbulence in a thick layer $\left(h_{\mathrm{t}} / l_{\mathrm{f}} \approx 0.78\right)$ supports the inverse energy cascade, as evidenced by the $S_{3 \mathrm{~L}}$ result and also by the generation of the strong spectral condensate fed by the cascade. Similar results are obtained in even thicker layers, up to $h_{\mathrm{t}}=12 \mathrm{~mm}$, or $h / l_{\mathrm{f}} \approx 1.3$.
Strong spectral condensates, the energy of which constitutes above $90 \%$ of the total flow energy, are observed in those cases.

After the development of the condensate, particle streaks are almost planar, at least in the statistical sense (Fig. 1d). Thus, there must be a mechanism through which the vortex secures its energy supply by suppressing vertical motions and enforcing planarity. To investigate the effect of a large flow on 3D turbulent motion, we perform experiments in a single layer of electrolyte, $h=10 \mathrm{~mm}$, with a much larger boundary, $L=300 \mathrm{~mm}$, to avoid spectral condensation. In this case the large-scale vortex is imposed externally. The timeline of the experiment is shown schematically in Fig. 2a. First, turbulence is excited. Then a large vortex $(150 \mathrm{~mm}$ diameter) is imposed on it by placing a magnetic dipole $2 \mathrm{~mm}$ above the free surface. After the large magnet is removed, the vortex decays, while turbulence continues to be forced. As the magnet blocks the view, the measurements are performed during the turbulence stage and during the decay of the vortex.

Figure $2 \mathrm{~b}$ shows the profiles $\left\langle V_{z}\right\rangle_{\mathrm{rms}}(z)$ of the vertical velocity fluctuations, measured using defocusing PIV. Before the vortex is imposed, vertical fluctuations are high, $\left\langle V_{z}\right\rangle_{\mathrm{rms}} \approx 1.6 \mathrm{~mm} \mathrm{~s}^{-1}$. When the vortex is present, they are reduced by a factor of about four, down to $\left\langle V_{z}\right\rangle_{\mathrm{rms}} \approx 0.4 \mathrm{~mm} \mathrm{~s}^{-1}$. This is also confirmed by the direct visualization of the flow in the vertical $z-y$ plane. Particle streaks are shown in Fig. 2c-e. Strong vertical eddies are seen in the turbulence stage, before the vortex is imposed (Fig. 2c). Shortly after the large magnet is removed, at $t-t_{0}=1 \mathrm{~s}$, the streaks show no vertical excursions. As the large vortex decays, the $3 \mathrm{D}$ eddies start to reappear near the bottom (Fig. 2e).

We also study the statistics of the horizontal velocities. The kinetic energy spectra and the third-order moments are shown in Fig. $2 \mathrm{f}-\mathrm{h}$. For turbulence without the vortex, the spectrum is substantially flatter than $k^{-5 / 3}$ (Fig. 2f). After the large vortex is imposed, the spectrum shows a strong peak at low wavenumbers. However, the mean subtraction recovers the $k^{-5 / 3} 2 \mathrm{D}$ spectrum, as shown in Fig. 2g. The third-order moment undergoes an even more pronounced change after the imposition of the large vortex: $S_{3 \mathrm{~L}}$ computed after the mean subtraction is much larger than during the turbulence stage, and it is a positive linear function of $r$, Fig. 2h, just as in the case of the double-layer experiment. Thus the imposed flow enforces planarity and strongly enhances the inverse energy flux.

The strong suppression of vertical eddies in the presence of an imposed flow must be due to the vertical shear $\Omega_{\mathrm{LS}}=\mathrm{d}\left\langle V_{\mathrm{h}}\right\rangle / \mathrm{d} z$, which destroys vertical eddies for which the inverse turnover time is less than $\Omega_{\text {LS }}$. This shear is obtained from the $z$-profiles of the horizontal velocity measured using defocusing PIV. In a single-layer experiment, the averaged shear of the horizontal velocity due to the presence of the strong imposed vortex is $\Omega_{\mathrm{LS}} \approx 1.6 \mathrm{~s}^{-1}$. Such a shear is sufficient to suppress vertical eddies with inverse turnover times $\tau^{-1} \sim\left\langle V_{z}\right\rangle_{\mathrm{rms}} / h \approx 0.16 \mathrm{~s}^{-1}$. In the spectrally condensed turbulence, an inverse energy cascade is sustained in layers that are substantially thicker than are possible in unbounded turbulence. A possible reason for this is also the shear suppression of the $3 \mathrm{D}$ vortices. Here the vertical shear is lower, $\Omega_{\mathrm{LS}} \approx 0.5 \mathrm{~s}^{-1}$, yet it substantially exceeds the inverse turnover time for the force-scale vortices in the doublelayer experiment, $\tau^{-1} \sim\left\langle V_{z}\right\rangle_{\mathrm{rms}} / h \approx 0.06 \mathrm{~s}^{-1}$. One might think that the pronounced change in the flow field in the presence of the large vortex is due to the global fluid rotation. However, the Rossby numbers in the reported experiments are larger (especially in the double layer experiment, $R o>3$ ) than those at which quasi-2D flow properties were observed in rotating tanks $(R o<0.4$; ref. 16).

The suppression of vertical motions by the shear flow and the onset of the inverse cascade observed in this experiment may be relevant for many natural and engineering applications. In the solar tachocline $^{17}$, a thin layer between the radiative interior and the outer convective zone, turbulence is expected to be $2 \mathrm{D}$, despite being excited by radial convection ${ }^{17}$. Turbulence suppression by 

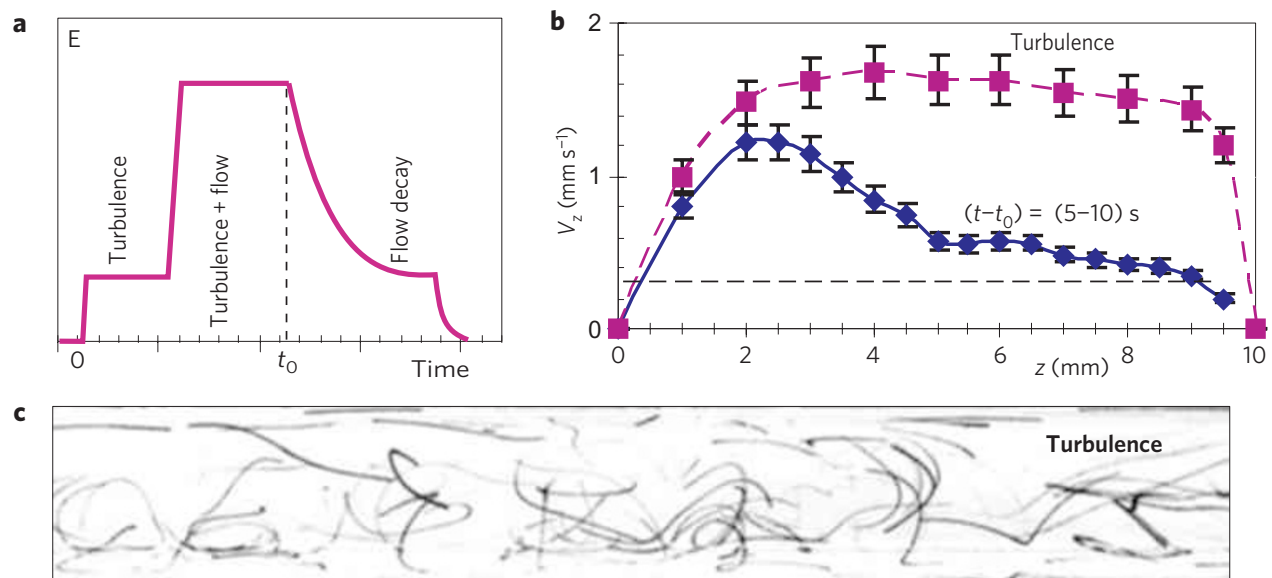

d
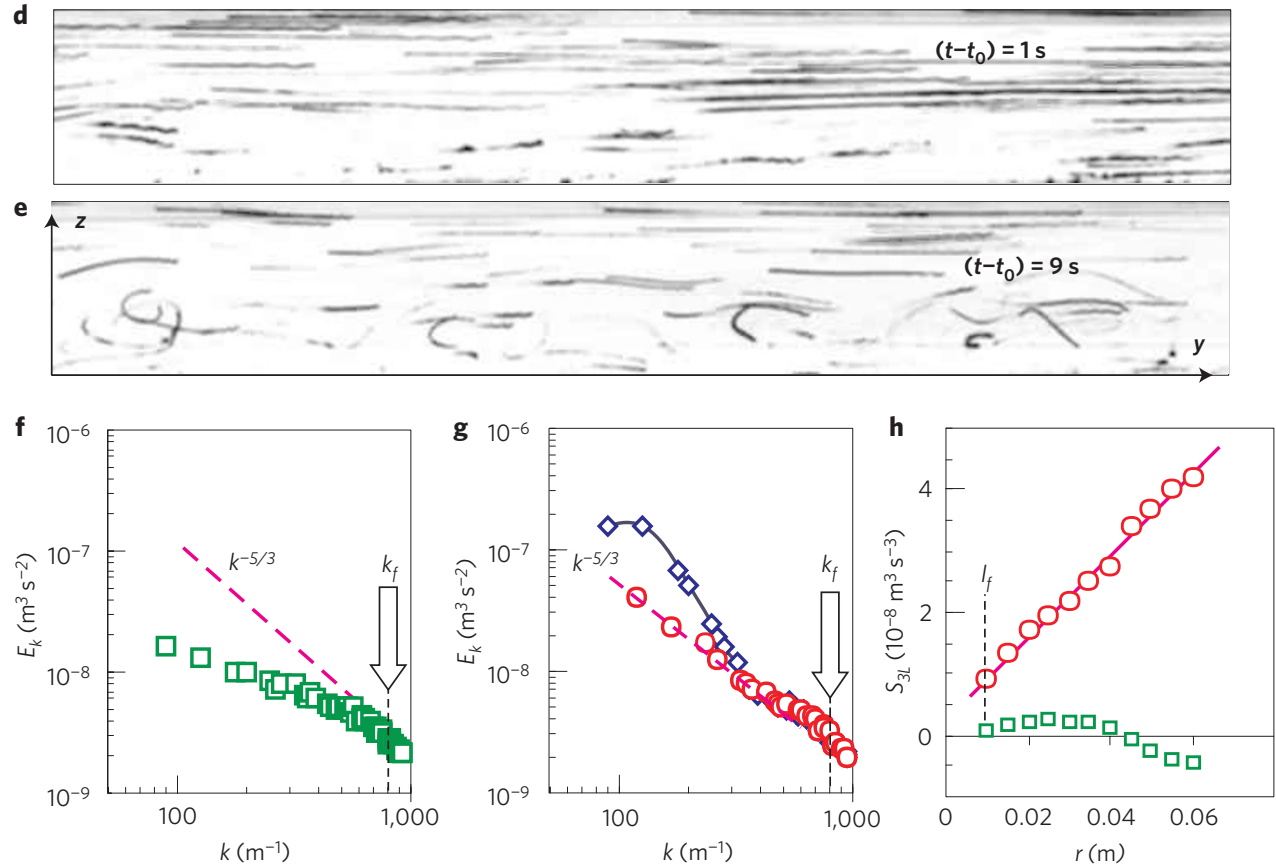

Figure 2 | Effects of externally imposed large-scale flow on turbulence in a single fluid layer (thickness $h=10 \mathrm{~mm}$, forcing scale $I_{\mathrm{f}}=8 \mathrm{~mm}$ ). The size of the horizontal plane box is $300 \mathrm{~mm}$. a, The time line of the experiment: turbulence forcing is switched on, then, $100 \mathrm{~s}$ later, a large scale vortex (150 mm diameter) is imposed on top of the turbulence. The vortex forcing is then removed at $t_{0}$. The large vortex decays, while the turbulence is still forced.

b, Measurements using the defocusing PIV technique show that vertical velocity fluctuations are substantially reduced by the large-scale vortex . Error bars correspond to the $10 \%$ instrumental accuracy of the technique. c, Streaks of seeding particles filmed in the vertical (z-y) cross-section show strong 3D motion before the large-scale vortex is imposed (exposure time is $2 \mathrm{~s}$ ). d, Particle streaks filmed during the decay of the large-scale vortex (exposure time is $1 \mathrm{~s}$ ) show that vertical motions are suppressed. e, As the vortex decays further, 3D eddies re-emerge from the bottom of the cell. $\mathbf{f}$, The spectrum of horizontal velocities is flat in turbulence. $\mathbf{g}$, During the decay of the large vortex the spectrum is dominated by the low-k spectral feature (open diamonds). The subtraction of the mean flow recovers the $k^{-5 / 3}$ cascade range (open circles). $\mathbf{h}$, The third-order structure function $S_{3 L}$ computed after the mean subtraction is a positive linear function of the separation distance (open circles). $S_{3 L}$ in the turbulence stage is much smaller (open squares).

the shear in the tachocline has been considered theoretically ${ }^{18}$. Present results indicate that only one velocity component may be strongly suppressed, making the turbulence $2 \mathrm{D}$. Another interesting example is the wavenumber spectrum of winds in the Earth atmosphere measured near the tropopause ${ }^{2}$, which shows $E(k) \sim$ $k^{-5 / 3}$ in the mesoscale range $(10-500 \mathrm{~km})$ and a strong peak at the planetary scale of $10^{4} \mathrm{~km}$. Numerous hypotheses have been proposed to explain the mesoscale spectrum, with most arguments centred on the direct versus the inverse energy cascade ${ }^{2,19-23}$. The shape of the spectrum alone cannot resolve this issue because both the 3D Kolmogorov direct cascade and the 2D Kraichnan inverse cascade predict $E(k) \sim k^{-5 / 3}$. Direct processing of atmospheric data gave $S_{3 \mathrm{~L}}(r)<0$ for some range of $r$ in the mesoscales, thus favouring the direct cascade hypothesis ${ }^{24}$. However, the subtraction of the mean flows, necessary for the correct flux evaluation, has not been done for the wind data. This leaves the question about the source of the mesoscale energy unresolved. Estimates of the vertical shear due to the planetary scale flow ${ }^{2}$, represented by the spectral peak at $10^{4} \mathrm{~km}$, show that the shear suppression criterion can be satisfied for small-scale eddies with sizes less than $10 \mathrm{~km}$. Thus, it is possible that the suppression of $3 \mathrm{D}$ vertical eddies induces an inverse energy cascade through the mesoscales in the Earth atmosphere. Moreover, our results may be relevant not only for thin layers but also for boundary layer flows with turbulence generated by surface roughness, convection or other sources. It may also shed light on the nature of velocity correlations at horizontal distances far exceeding the distance from the ground, which is important for wind farms and in solving many other problems. 
Received 25 June 2010; accepted 17 December 2010; published online 6 February 2011

\section{References}

1. Lilly, D. K. \& Petersen, E. L. Aircraft measurements of atmospheric kinetic energy spectra. Tellus 35A, 379-382 (1983).

2. Nastrom, G. D., Gage, K. S. \& Jasperson, W. H. The kinetic energy spectrum of large- and mesoscale atmospheric processes. Nature 310, 36-38 (1984).

3. Kraichnan, R. H. Inertial ranges in two-dimensional turbulence. Phys. Fluids 10, 1417-1423 (1967).

4. Sommeria, J. Experimental study of the two-dimensional inverse energy cascade in a square box. J. Fluid Mech. 170, 139-168 (1986).

5. Paret, J. \& Tabeling, P. Intermittency in the two-dimensional inverse cascade of energy: Experimental observations. Phys. Fluids 10, 3126-3136 (1998).

6. Shats, M., Xia, H. \& Punzmann, H. Spectral condensation of turbulence in plasmas and fluids and its role in low-to-high phase transitions in toroidal plasma. Phys. Rev. E 71, 046409 (2005)

7. Vorobieff, P., Rivera, M. \& Ecke, R. E. Soap film flows: Statistics of two-dimensional turbulence. Phys. Fluids 11, 2167-2177 (1999).

8. Belmonte, A. et al. Velocity fluctuations in a turbulent soap film: The third moment in two dimensions. Phys. Fluids 11, 1196-1200 (1999).

9. Smith, L. M., Chasnov, J. R. \& Fabian, W. Crossover from two- to three-dimensional turbulence. Phys. Rev. Lett. 77, 2467-2470 (1996).

10. Celani, A., Musacchio, S. \& Vincenzi, D. Turbulence in more than two and less than three dimensions. Phys. Rev. Lett. 104, 184506 (2010).

11. Shats, M. G., Xia, H., Punzmann, H. \& Falkovich, G. Suppression of turbulence by self-generated and imposed mean flows. Phys. Rev. Lett. 99, 164502 (2007).

12. Xia, H., Punzmann, H., Falkovich, G. \& Shats, M. Turbulence-condensate interaction in two dimensions. Phys. Rev. Lett. 101, 194504 (2008).

13. Xia, H., Shats, M. \& Falkovich, G. Spectrally condensed turbulence in thin layers. Phys. Fluids 21, 125101 (2009).

14. Willert, C. E. \& Gharib, M. Three-dimensional particle imaging with a single camera. Exp. Fluids 12, 353-358 (1992).

15. Chertkov, M., Connaughton, C., Kolokolov, I. \& Lebedev, V. Dynamics of energy condensation in two-dimensional turbulence. Phys. Rev. Lett. 99, 084501 (2007).
16. Hopfinger, E. J., Browand, F. K. \& Gagne, Y. Turbulence and waves in a rotating tank. J. Fluid Mech. 125, 505-534 (1982).

17. Spiegel, E. A. \& Zahn, J-P. The solar tachocline. Astron. Astrophys. 265, 106-114 (1992)

18. Kim, E-J. Self-consistent theory of turbulent transport in the solar tachocline. Astron. Astrophys. 441, 763-772 (2005).

19. Frisch, U. Turbulence: The Legacy of A.N. Kolmogorov (Cambridge Univ. Press, 1995).

20. Gage, K. S. \& Nastrom, G. D. Theoretical interpretation of atmospheric wavenumber spectra of wind and temperature observed by commercial aircraft during GASP. J. Atmos. Sci. 43, 729-740 (1986).

21. Lilly, D. K. Two-dimensional turbulence generated by energy sources at two scales. J. Atmos. Sci 46, 2026-2030 (1989).

22. Falkovich, G. Inverse cascade and wave condensate in mesoscale atmospheric turbulence. Phys. Rev. Lett. 69, 3173-3176 (1992).

23. Smith, L. \& Yakhot, V. Finite-size effects in forced two-dimensional turbulence. J. Fluid Mech. 274, 115-138 (1994).

24. Cho, J. Y. N. \& Lindborg, E. Horizontal velocity structure functions in the upper troposphere and lower stratosphere. J. Geophys. Res. 106, 10223-10232 (2001).

\section{Acknowledgements}

This work was supported by the Australian Research Council's Discovery Projects funding scheme (DP0881544) and by the Minerva Foundation and the Israeli Science Foundation.

\section{Author contributions}

H.X., D.B. and M.S. designed and performed experiments; H.X. and D.B. analysed the data. M.S. and G.F. wrote the paper. All authors discussed and edited the manuscript.

\section{Additional information}

The authors declare no competing financial interests. Reprints and permissions information is available online at http://npg.nature.com/reprintsandpermissions. Correspondence and requests for materials should be addressed to M.S. 